ORIGINAL RESEARCH

\title{
A study of prevalence of silent coronary artery disease in asymptomatic type II diabetes mellitus \\ Moogaambiga $S^{1, *}$, Kirubhakaran $\mathrm{K}^{1}$, Lavanya Devi $\mathrm{P}^{1}$ and Santhosh $\mathrm{P}^{1}$
}

${ }^{1}$ Department of General Medicine, Vinayaka Mission's Kirupananda Variyar Medical College and Hospital, Salem, Tamil Nadu- 636308, India

\begin{abstract}
Background: Cardiovascular disease is a major cause of morbidity and mortality in diabetics. However diabetic patients do not present with typical anginal symptoms or may be even asymptomatic and silent coronary artery disease (CAD) is prevalent in diabetics. Moreover silent CAD is not different from symptomatic CAD with respect to prognosis and adverse effects.
\end{abstract}

Aim: The study was done to demonstrate the prevalence of silent myocardial ischemic changes in asymptomatic type 2 diabetes mellitus patients with normal resting ECG by doing a stress exercise test.

Methodology: This descriptive study was done in 100 patients with type 2 diabetes more than 2 years who did not have any history of cardiovascular disease or symptoms. Detailed history was taken and investigations such as HbA1c, fasting and postprandial blood sugar, serum creatinine, urine examination were done. Resting ECG, Echocardiogram was found to be normal and they were subjected to a treadmill test.

Results: Among the 100 participants 18 persons (18\%) had positive ECG findings in treadmill test. A positive association was found between the duration of diabetes and prevalence of positive treadmill test.

Conclusion: There is significant prevalence of silent CAD in diabetic patients and they tend to present with advanced disease at presentation and have poorer prognosis compared to non diabetic population. Hence it is necessary to screen early for silent CAD in diabetics to improve disease outcomes.

Keywords: diabetes; silent CAD; treadmill test

*Corresponding author: Dr. Moogaambiga S, Department of General Medicine, Vinayaka Mission's Kirupananda Variyar Medical College and Hospital, Salem, Tamil Nadu- 636308, India. Mobile: 9842459677; E-mail: mooks.stanlean@gmail.com.

Received 16 October 2020; Revised 2 December 2020; Accepted 12 December 2020; Published 30 December 2020

Citation: Moogaambiga S, Kirubhakaran K, Devi PL, Santhosh P. A study of prevalence of silent coronary artery disease in asymptomatic type II diabetes mellitus. J Med Sci Res. 2021; 9(1):1-4. DOI: http://dx.doi.org/10.17727/JMSR.2021/9-1
Copyright: (C) 2021 Moogaambiga S et al. Published by KIMS Foundation and Research Center. This is an open-access article distributed under the terms of the Creative Commons Attribution License, which permits unrestricted use, distribution, and reproduction in any medium, provided the original author and source are credited. 


\section{Introduction}

The prevalence of diabetes mellitus has increased exponentially over the past two decades. The International Diabetic Federation predicts that 629 million people would be affected by diabetes by 2045 [1]. The metabolic dysregulation associated with diabetes causes secondary pathophysiological changes in several organ systems. Cardiovascular disease is a major complication and main cause of morbidity and mortality in more than $50 \%$ of all diabetic patients [2]. Cardiovascular risk factors like hypertension, dyslipidemia and obesity are more common in patients with diabetes. Moreover factors in diabetes like increased oxidative stress, increased coagulability, endothelial dysfunction have a direct role in the pathogenesis of coronary artery disease (CAD) [3]. These mechanisms that are unique to diabetes contribute to the pathophysiology of cardiac disease in addition to and independent of other risk factors like hypertension and dyslipidemia [4]. Individuals with diabetes have a higher risk of atherosclerotic burden and lesser coronary artery lumen diameter than a non diabetic patient. Angina is the main symptom of ischemic cardiovascular disease. However diabetic patients do not present with typical anginal symptoms or may be even asymptomatic and silent CAD disease is common in diabetics [5]. Episodes of transient myocardial ischemia may be silent, though ST,T changes may be seen in ECG during treadmill tests. In high risk diabetic patients, over the last years it has been well recognized that silent CAD is not different from symptomatic CAD with respect to prognosis and adverse effects [6]. Our study was done to demonstrate the prevalence of silent myocardial ischemic changes in asymptomatic type 2 DM patients with normal resting ECG by doing a stress exercise test.

\section{Methodology}

This is a descriptive study done at Vinayaga Missions Medical College, Salem with 100 type 2 diabetes patients from both outpatient as well as inpatient departments.

Type 2 diabetes patients with duration of diabetes more than two years who did not have any complaints related to cardiovascular disease were included. Patients between ages of 30 to 70 years were included.
Patients with duration of diabetes less than 2 years and those with history of angina, myocardial infarction, heart failure, arrhythmias were excluded. Known cases of cardiomyopathy, previous coronary artery bypass surgery and other chronic diseases like chronic kidney disease, decompensated liver disease were excluded. Patients with previous cerebrovascular accident were also excluded.

A detailed history was taken regarding diabetes and other symptoms followed by clinical examination. Then the following investigations $\mathrm{HbA1c}$, fasting and postprandial blood sugar, serum creatinine, urine examination were done. Patients were diagnosed to be diabetic if the fasting plasma glucose $>126 \mathrm{mg} /$ $\mathrm{dl}$ or postprandial blood sugar $>200 \mathrm{mg} / \mathrm{dl}$ [7]. In a patient with classical symptoms of hyperglycaemia a random blood plasma glucose $>200 \mathrm{mg} / \mathrm{dl}$ was considered diagnostic. Patients were also clinically tested for autonomic neuropathy using cardiac autonomic reflex tests. Patients were then subjected to echocardiogram and treadmill test. ECG was taken for all the patients. The calculation and data analysis were done by SPSS software version 21.0.

\section{Results}

Majority of the participants (33\%) were in the age group 51-60 years, followed by 29\% in 41-50 years and $19 \%$ in $31-40$ years and $61-70$ years. $55 \%$ of our patients were males and $45 \%$ were females. Among the study participants duration of diabetes was more than 10 years in $14 \%$, duration was 5-10 years in $50 \%$ and less than 5 years in $36 \% .6 \%$ of the patients were also found to be hypertensive while the remaining $94 \%$ of the patients were normotensive. Majority of the study participants (62\%) were obese, having a body mass index (BMI) $>25$, while 9\% were overweight with BMI between 23-24.9 and $29 \%$ had normal BMI. $18 \%$ of the patients were requiring insulin for glycemic control whereas the remaining were on oral hypoglycemic drugs. 10\% of the patients had clinical evidence of autonomic neuropathy. Among the 100 participants 18 persons (18\%) had positive ECG findings in treadmill test (Table 1). Majority of the participants (62\%) were having uncontrolled diabetes with $\mathrm{HbA} 1 \mathrm{c}$ levels more than 7.5 , while $38 \%$ were having the diabetes under control. We analysed the association between a positive treadmill test and duration of diabetes using chi square test. $36 \%$ participants had diabetes for short duration ( $<5$ years) and 64\% 
had diabetes for longer duration ( $>5$ years) (Table 2). Higher proportion of the patients with diabetes mellitus (DM) having longer duration of the disease tested positive than the ones with short duration of diabetes and the association was found to be statistically significant $(\mathrm{p}=0.015)$. We also analysed the association between diabetes control status and the TMT findings. Higher proportion of the patients with uncontrolled DM status tested positive than the ones with controlled diabetes and the association was found to be statistically significant $(\mathrm{p}=0.009)$ (Table 3). Among the 18 patients who tested positive for treadmill test 10 patients had clinical findings of autonomic neuropathy.

Table 1: Distribution of participants according to TMT findings $(\mathrm{N}=100)$.

\begin{tabular}{|ccc|}
\hline TMT & Frequency $N=100$ & Percent (\%) \\
\hline Positive & 18 & 18 \\
Negative & 82 & 82 \\
\hline
\end{tabular}

Table 2: Association between the duration of diabetes and TMT findings.

\begin{tabular}{|lccc|}
\hline \multirow{2}{*}{ Duration } & \multicolumn{2}{c}{ TMT } & Pvalue \\
\cline { 2 - 3 } & Positive & Negative & \\
\hline $\begin{array}{l}\text { Short duration } \\
\text { ( }<5 \text { years })\end{array}$ & 2 & 34 & \\
$\begin{array}{l}\text { Long duration } \\
(>5 \text { years })\end{array}$ & 16 & 48 & 0.015 \\
Total & 18 & 82 & \\
\hline
\end{tabular}

Table 3: Association between Diabetes control status and TMT findings.

\begin{tabular}{|lccc|}
\hline \multirow{2}{*}{ HbA1c } & \multicolumn{2}{c}{ TMT } & \multirow{2}{*}{ Pvalue } \\
\cline { 2 - 3 } & Positive & Negative & \\
\hline Controlled DM & 2 & 36 & \\
Uncontrolled DM & 16 & 46 & 0.009 \\
Total & 18 & 82 & \\
\hline
\end{tabular}

\section{Discussion}

This study of prevalence of silent coronary artery disease in asymptomatic type 2 diabetes mellitus (T2DM) was conducted in Vinayaka Missions Medical College, Salem in the period of January 2018 to August 2020. Total of 100 patients who attended both inpatient department and outpatient department were included in this study and all underwent initial evaluation with history taking, detailed physical examination and also routine investigations.

In our study, there is no obvious sex difference observed in the prevalence of T2DM (55 males and 45 females). And this study resembles majority of studies conducted elsewhere that show there is no sex difference in the prevalence of T2DM.

None of our patients showed any cardiac abnormality at rest (Resting ECG and 2D ECHO was found to be within normal limits). They did not have any cardiovascular symptoms either. TMT was undertaken in all the 100 patients, out of whom 18 patients showed inducible ischemia without anginal symptoms. This is similar to the findings of the study in new-onset middle aged type 2 diabetic patients to see the prevalence of silent myocardial ischemia done [5]. In that study, 19 patients showed (17.1\%) inducible ischemia in treadmill test.

Majority of the patients in our study (81\%) were more than 40 years of age.

In this study, $36 \%$ patients had diabetes $<5$ years of duration. $50 \%$ patients had diabetes of 5-10 year duration and $14 \%$ patients had diabetes $>10$ years of duration.

Among 18 patients who tested positive in treadmill test, higher proportion of them had longer duration of the disease of $>5 \mathrm{yrs}$. The longer the duration of diabetes, the increased chances of getting a positive treadmill test. This reiterates the point that the risk of coronary heart disease increases with the duration of diabetes even after adjustment for age, sex and other risk factors [8].

Among these 18 patients in our study (18\%), those showed inducible ischemia in exercise test, 17 patients (94.5\%) were $>50$ years of age and remaining one patient $(5.5 \%)$ was $<50$ years which shows that myocardial ischemia develops as age advances even in the absence of classical and prominent symptoms in diabetes.

All these 18 patients, who had exercise-inducible ischemia in TMT showed ST depression of $>1 \mathrm{~mm}$ without angina symptoms

Our study shows BMI of $<25$ in 38 patients (38\%) and $>25$ in 62 patients (62\%). Body mass index is 
very strongly associated with diabetes and insulin resistance [9]. Obesity which is actually a major risk factor for developing diabetes is also a major risk factor for coronary artery disease.

Among these 18 patients who showed positive TMT, HbA1c value was more than 7.5 in 16 patients $(90 \%)$ and less than 7.5 in 2 patients (10\%) which clearly shows there is a positive association between uncontrolled diabetes and increased cardiovascular risk and also coincides with many studies which shows positive correlation between myocardial ischemia and elevated $\mathrm{HbA1c}$.

In our study, autonomic neuropathy was detected in 10 out of 18 asymptomatic diabetics those showed inducible ischemia on TMT. In diabetic patients, autonomic neuropathy is responsible for absent anginal pain during ischemic episodes. The presence of cardiac autonomic neuropathy is a strong predictor of mortality in diabetic patients [10]. In a recent study to correlate the incidence of autonomic neuropathy and silent myocardial ischemia in type 2 diabetic patients by "AU Alhayali J.MT" and "Alnuemi A.A", silent myocardial ischemia was detected in 12 (26.6\%) diabetic patients in general. 8 (66.7\%) of them had autonomic neuropathy [11]. It shows us the necessity of screening of diabetic patients with autonomic neuropathy.

Angina occurring due to ischemia serves as a clinical warning for patients developing obstructive CAD [12]. However silent ischemia is more prevalent in diabetics and diabetic patients with silent ischemia are often asymptomatic and are diagnosed later with progressed late stage of CAD. This would explain the findings seen in some angiographic studies where diabetic patients had more advanced CAD even at the time of diagnosis $[13,14]$. This is sadly responsible for higher rates of CAD related mortality and morbidity in diabetics.

\section{Conclusion}

$18 \%$ (18 out of 100) of asymptomatic diabetics showed positive test on TMT without symptoms of angina though none of them had resting ECG changes and 2D ECHO cardiac abnormalities. This high prevalence of silent CAD in diabetics justifies the need for early screening for CAD in diabetic patients. Most of the treadmill positive diabetes patients were having HbA1c of more than $7.5 \%$. Statistically significant association was found between the duration of diabetes and prevalence of asymptomatic CAD. We can infer that both duration of diabetes as well as adequate glycemic control are vital factors determining the severity of cardiovascular complications. Considering the increased frequency of CAD in diabetics, silent progression of the disease and presence of advanced disease at presentation and poorer prognosis of CAD in diabetics, it would be justified to screen asymptomatic diabetic patients for silent CAD.

\section{Conflicts of interest}

Authors declare no conflicts of interest.

\section{References}

[1] IDF Diabetes Atlas, 8th ed. International diabetes federation; 2017. Accessed from: www.diabetesatlas.org.

[2] Dei Cas A, Khan SS, Butler J, Mentz RJ, Bonow RO, et al. Impact of diabetes on epidemiology,treatment and outcomes of patients with heart failure. JACC Heart Fail. 2015; 3(2):136145.

[3] Matheus AS, Tannus LR, Cobas RA, Palma CC, Negrato CA, Gomes MB. Impact of diabetes on cardiovascular disease: an update. Int J Hyprtens. 2013; 2013:653789.

[4] Dokken BB. The pathophysiology of cardiovascular disease and diabetes: beyond blood pressure and lipids. Diabetes Spectrum 2008; 21(3):160-165.

[5] Fornengo P, Bosio A, Epifani G, Pallisco O, Mancuso A, et al. Prevalence of silent myocardial ischemia in new onset middle aged type 2 diabetic patients without other cardiovascular risk factors. Diabet Med. 2006; 23(7):775-779.

[6] Haffner SM, Lehto S, Ronnema T, Pyoralak, Laakso M. Mortality from CHD in subjects with type II DM and in nondiabetic subjects with and without prior MIN. Eng J Med. 1998; 339(4):229-232.

[7] American Diabetes Association. Classification and diagnosis of diabetes: Standards of medical care in diabetes-2020. Diabetes Care. 2020; 43(Suppl 1):S14-S31.

[8] Fox CS, Sullivan L, D’Agostino RB, Wilson PWF, Framingham Heart Study. The significant effect of diabetes duration on coronary heart disease mortality. Diabetes care 2004; 27(3):704-708.

[9] Al-Goblan AS, A Al-Alfi MA, Khan MZ. Mechanisms linking diabetes mellitus and obesity. Diabetes Metab Syndr Obes. 2014; 7:587-591.

[10] Agashe S, Petak S. Cardiac autonomic neuropathy in diabetes mellitus. Methodist Debakey Cardiovasc J. 2018; 14(4): 251256.

[11] Alhayali A, Alnuemi A. Autonomic neuropathy and silent myocardial ischemia in type II diabetic patients. Middle East Journal of Emergency Medicine; 2006; 6(1):25-31.

[12] Boras J, Brkljacic N, Ljubicic A, Ljubic S. Silent ischemia and diabetes mellitus. Diabetologia Croatica. 2010; 39(2):5765.

[13] Fazzini PF, Prati PL, Rovelli F, Antoniucci D, Menghini F, et al. Epidemiology of silent myocardial ischemia in asymptomatic middle aged men. Am J Cardiol. 1993; 72(18):1383-1388.

[14] Koistinen MJ. Prevalence of asymptomatic myocardial ischemia in diabetic subjects. BMJ. 1990; 301(6743):92-95. 